\title{
SPECTROPHOTOMETRIC POPULATION SYNTHESIS OF EARLY TYPE GALAXIES
}

\section{A. VAZDEKIS}

Instituto de Astrofísica de Canarias, E-38200 La Laguna, Tenerife, Spain

R. PELETIER

Kapteyn Institute, Groningen, the Netherlands

AND

E. CASUSO AND J. BECKMAN

Instituto de Astrofísica de Canarias, E-38200 La Laguna, Tenerife, Spain

We have developed a new stellar population synthesis model for calculating colours and absorption line indices in early type galaxies. This model can work either for single-age stellar populations or in an evolutionary scheme following the chemical evolution. The model is based on the isochrones of the Padova group and we have developed our own method of conversion to colours. Details can be found in Vazdekis A., Casuso E., Peletier R. \& Beckman J. (submitted, 1995). To test the model we have obtained accurate observations in many colours and line indices of the three standard galaxies: NGC 3379, NGC 4472 and NGC 4594.

In general we can find reasonably solutions for the galaxies, with $\mathrm{Z}>$ $\mathrm{Z}_{\odot}$ (Casuso et al., ApJ. 1995, In Press.) Fits are good for most colours and absorption line indices except for those of $\mathrm{Fe}$ and $\mathrm{Ca}$. Including $\alpha$ enhancement improves the fit for Fe, but worsens the fit for the $\mathrm{NaD}$ index. For an evolutionary scheme with a single constant IMF slope, in a closedbox approximation, the $\mathrm{Mg}_{2}$ index from the models always falls short of the observed values (Vazdekis et al. (1995). Finally, we obtain much better fits if we introduce a significant change in the IMF slope, favouring massive stars in the early stages of galactic evolution, and low-mass stars for the remaining time. 